\title{
Herpes simplex virus type II in a patient having periodic lateralized epileptiform discharges
}

Justin Chuang, MSIV ${ }^{1}$, Sona Shah, MD ${ }^{1}$

\section{Author Affiliation:}

1. Marshall University Joan C. Edwards School of Medicine, Huntington, West Virginia

The authors have no conflicts of interest to disclose.

\section{Corresponding Author:}

Justin Chuang, MSIV

Marshall University

Joan C. Edwards School of Medicine

Huntington, West Virginia

Email: chuang@marshall.edu 


\section{Abstract}

Herpes Simplex Encephalitis has been found to be one of the most common causes of fatal viral encephalitis. ${ }^{1}$ The mortality rate in untreated patients has been found to be as high as $70 \%$ and thus correct management and early diagnosis are important for good prognosis and crucial for predicting outcome. ${ }^{1,2}$ In patients with Herpes Simplex Virus (HSV), periodic lateralized epileptic form discharges (PLEDs) are considered a specific finding on electroencephalogram (EEG). PLEDs are usually seen in the context of destructive structural lesions involving the cortex; other causes include acute ischemic stroke and less commonly, tumors. Finding of PLEDs has been linked to epileptic seizures, including status epilepticus. ${ }^{3}$

We report a case of HSV type 2 infection and PLEDs. Unlike HSV type 1 infection, this is not a common presentation.

\section{Keywords}

HSV2, periodic lateralized epileptiform discharges, PLEDS, HSV 2 PLEDS, Herpes simplex virus type 2 PLEDS, PLEDS HSV, HSV-2 encephalitis PLEDS

\section{Case Report}

A 62-year-old man was transferred to our facility for higher level of care after being admitted to outside hospital [OSH] for four days with shortness of breath and cough with yellow/greenish sputum without hemostasis. There was concern for recurrent pneumonia with temperature of 99.2 and WBC count of 13. OSH noted patient four days ago being lethargic and agitated along with history of single seizure like event with eyes rolling back with loss of consciousness lasting for 3 minutes. BP at that time was 186.123. OSH MRI brain WO was read as punctate foci of restricted diffusion with corresponding low signal in ADC sequence in left parietal lobe. CT chest results were considered consistent with pneumonia with plural effusion. Additional mural thrombi in the proximal abdominal aorta were found without dissection or aneurysm. Patient was transferred and treated for pneumonia. Secondary stroke prevention treatment was also given.

His PMH was remarkable for throat cancer with s/p chemotherapy and radiation, progressive dysphagia, multiple admission for pneumonia in past 6 weeks with steroids administration, COPD, HT, HLP, DM, cachexia, CAD stent 2007, chronic pancreatitis and three pancreatic surgeries with right eye being blind. Social history was remarkable for continued smoking and ETOH use with possible concern of ETOH abuse. Allergy history was positive for penicillin allergy.

On the day of admission neurology consult examination suggested that patient was alert and oriented times 2 and disoriented in the time with intact language, speech, cranial nerves, motor, sensory exam and intact deep tendon reflexes with down going planters and normal coordination. At that time history of agitation, confusion and acting differently was noted but OSH of seizure was not mentioned in the note. OSH MRI was personally reviewed by neurologist and secondary stroke prevention was recommended and treated per standard of care. On admission day 2, CT head without contrast did not reveal any acute changes other than chronic microvascular changes seen in MRI at OSH; neurology note mentioned seizures and EEG was ordered and obtained 
with exam unchanged except patient was found to be disoriented. The EEG was interpreted by neurophysiologist (with one year fellowship) as technically inadequate due to abundant EMG, electrode and $60 \mathrm{~Hz}$ artifacts with neurology management kept unchanged. On day 3 after admission, patient was found to be agitated overnight; neuro exam was performed after sedation and patient did not follow the commands.

On day 4 after admission, patient was observed to have generalized tonic clonic [GTC] seizure which led to treatment with Ativan. The start of continuous long term EEG monitoring [cEEG] led to involvement of epileptologist (with 2 years of formal training and 9 years of post-training experience). At request of neurophysiologist, epileptologist reviewed routine EEG done on Day 2 and felt there were underlying epileptiform abnormalities in the right hemisphere over and above myogenic and movement artifacts. Stat and ongoing review of cEEG over next 48 hours led to discovery of periodic lateralized epileptiform discharges [PLEDs] at lead P4 and T6 with frequency of 1 to $1.5 \mathrm{~Hz}$. The activation was performed at the onset of cEEG without any change in the abnormalities with photic activation. Patient was not able to perform hyperventilation due to abnormal mental status. Patient was also loaded with levetiracetam 1 gram followed by q 12 IVPB dose after this grandma seizure.

On day 5, neurology exam by stroke neurologist suggested that patient was nonverbal, unable to follow commands and displayed flaccid quadruparesis. On day 5, patient desaturated despite being on BiPAP earlier that day, and was intubated. There was lack of subclinical seizures in cEEG during desaturation period. cEEG had additional infrequent F7, T3 PLEDs. Levetiracetam was increased to q 8 hourly and IV sodium valproate was loaded at $20 \mathrm{mg} / \mathrm{kg}$ followed by maintenance scheduled dosing. Same day LP showed zero RBC, 1 WBC, glucose of 108 and protein high at 48 with clear fluid.

On day 6, patient remained intubated but did not need continuous sedative drips. He weakly responded to painful stimuli on all four extremities with new development of hypernatremia in range of 163. Neuro-status remained unchanged for following days until day 9 when he weakly followed command of wiggling his toes, more on right side than left. Sodium on day 8 returned to normal range with same day MRI brain WWO revealed restricted diffusion with increase in FLAIR and T2 signal intensities in both temporal lobes, right more than left, with gyriform enhancement in the region. This along with findings of PLED led to empirical onset of start of Acyclovir which was adjusted by creatinine clearance periodically. He was also given Cefepime 1 gram q 8 .

CSF results became available as positive HSV II by PCR on day 7. CSF studies showed negative cryptococcal antigen, nonreactive VDRL, negative for enterovirus, negative AFB and fungal culture with no growth in gram stain. WBC continued trending upwards up until day 10. Urine culture was negative as were two sets of blood culture. Sputum culture revealed usual expected flora. He remained intermittently febrile until day 11 with highest temperature of 103.3 on day 6. Infectious disease (ID) consult after CSF HSV results recommended continuation of acyclovir for 21 days with notation that CSF with HSV II could be quite unremarkable. Due to severe bilateral cerebral involvement, mortality could be up to 20 to $30 \%$. Long term prognosis was considered poor and lack of meaningful recovery was feared. The recovery of his original 
personality was considered unlikely and he was likely to remain with significant memory impairment.

Patient remained intubated until day 12, extubated, desaturated and reintubated on day 14. Family chose not to consider PEG or Trach. He was given comfort care, extubated and passed away on day 16.

\section{Discussion}

This uncommon presentation of HSV 2 infection led to delay in the detection. Also the association of HSV 2 with PLEDs is not well known.

A thorough search was conducted on Pubmed, Cochrane Library, and Google. Key words included HSV 2 Periodic Lateralized Epileptiform Discharges, PLEDS, HSV 2 PLEDS, herpes simplex virus type 2 PLEDS, PLEDS HSV, Periodic Lateralized Epileptiform Discharges HSV, herpes simplex encephalitis PLEDS, HSV-2 encephalitis PLEDS. There was only one article found that had a relationship between HSV2 and PLEDS as stated above. ${ }^{4}$ In addition to the online search, a thorough investigation through the entire Marshall Health System yielded negative results for patients that had both PLEDS and HSV-2 associated encephalitis. Hence, we are further supporting that this association has not been widely reported and is a rare finding.

PLEDs are an EEG signature of a dynamic pathophysiological state with underlying neuronal injury. $16 \%$ of cases of PLEDs present in single region are due to infection. However, this incidence goes up to $28 \%$ when two independent regions have independent PLEDs, as in our case. The PLEDs are known to be associated with seizures and a case can be made that patient could have been in subclinical status between day 2 and day 4 until continuous EEG was started. The prognosis of PLEDs is mainly dependent on the underlying pathology. 


\section{References}

1. Asnis D, Niazi N. Nondominant hemisphere encephalitis in patient with signs of viral meningitis. Emerging Infectious Diseases. 2009;15(8):1320-1321. doi:10.3201/eid1508.090466.

2. Domingues RB, Tsanaclis AM, Pannuti CS, Mayo MS, Lakeman FD. Evaluation of the range of clinical presentations of herpes simplex encephalitis by using polymerase chain reaction assay of cerebrospinal fluid samples. Clin Infec Dis. 1997;25:86-91.

3. Beneto A, Gomez E, Rubio P, Sobrino R, Esparza A, Gil M. et al. Periodical EEG pattern modifications in herpetic encephalitis treated with acyclovir. Rev Neurol. 1996;24:829-832.

4. Ansari M K, Jha S. Herpes simplex encephalitis in a patient having common variable immuno-deficiency. Ann Trop Med Public Health. 2010;3:30-32. 